\title{
Regulation of Tomato Prf by Pto-like Protein Kinases
}

\author{
Tatiana S. Mucyn, ${ }^{1,2}$ Ai-Jiuan Wu, ${ }^{1,2}$ Alexi L. Balmuth, ${ }^{1}$ Julia Maryam Arasteh, ${ }^{1}$ and John P. Rathjen ${ }^{1}$ \\ ${ }^{1}$ The Sainsbury Laboratory, Colney, Norwich NR4 7UH, U.K.; and ${ }^{2}$ Department of Biology, CB no. 3280, Coker Hall, \\ The University of North Carolina at Chapel Hill, Chapel Hill 27599-3280, U.S.A.
}

Submitted 31 July 2008. Accepted 14 December 2008.

\begin{abstract}
Tomato Prf encodes a nucleotide-binding domain shared by Apaf-1, certain R proteins, and CED-4 fused to C-terminal leucine-rich repeats (NBARC-LRR) protein that is required for bacterial immunity to Pseudomonas syringae and sensitivity to the organophosphate fenthion. The signaling pathways involve two highly related protein kinases. Pto kinase mediates direct recognition of the bacterial effector proteins AvrPto or AvrPtoB. Fen kinase is required for fenthion sensitivity and recognition of bacterial effectors related to AvrPtoB. The role of Pto and its association with Prf has been characterized but Fen is poorly described. We show that, similar to Pto, Fen requires N-myristoylation and kinase activity for signaling and interacts with the $\mathrm{N}$ terminal domain of Prf. Thus, the mechanisms of activation of Prf by the respective protein kinases are similar. Prf-Fen interaction is underlined by coregulatory mechanisms in which Prf negatively regulates Fen, most likely by controlling kinase activity. We further characterized negative regulation of Prf by Pto, and show that regulation is mediated by the previously described negative regulatory patch. Remarkably, the effectors released negative regulation of Prf in a manner dependent on Pto kinase activity. The data suggest a model in which Prf associates generally with Pto-like kinases in tightly regulated complexes, which are activated by effector-mediated disruption of negative regulation. Release of negative regulation may be a general feature of activation of NBARC-LRR proteins by cognate effectors.
\end{abstract}

Microbial plant pathogens are predominately extracellular and are perceived by the host on two levels (Chisholm et al. 2006). Characteristic, conserved molecules known as pathogenassociated molecular patterns (PAMPs) are ligands for receptors located in the plasma membrane, and elicit internal signal transduction pathways leading to immune responses. Second, detection of pathogen virulence molecules called effectors within the plant cell by resistance $(\mathrm{R})$ proteins leads to strong immune reactions, often including a cell death phenotype known as the hypersensitive response (HR). Effectors are secreted into host cells by pathogens as part of a strategy to manipulate host immunity. Thus, $\mathrm{R}$ proteins can be understood as an evolutionary response to pathogen virulence mechanisms.

Recognition of effector molecules by $\mathrm{R}$ proteins is highly specific (Jones and Dangl 2006). The simplest mode of interaction between effector and R components is direct. However,

Corresponding author: John Rathjen; Telephone: +44 1603 450405; Fax: +44 1603 450011; E-mail: john.rathjen@tsl.ac.uk

* The $\boldsymbol{e}$-Xtra logo stands for "electronic extra" and indicates that three supplementary figures are published online.
$\mathrm{R}$ proteins, which typically possess a nucleotide-binding domain shared by Apaf-1, certain R proteins, and CED-4 fused to C-terminal leucine-rich repeats (NBARC-LRR proteins), are often members of large molecular complexes and, in this case, other components of the complex can confer the interaction function. For example, the Arabidopsis thaliana protein RIN4 interacts with at least three pathogen effector molecules and is a member of separate complexes with the NBARC-LRR proteins RPS2 and RPM1 (Jones and Dangl 2006). Certain modifications of RIN4 by bacterial effectors can lead to activation of either R protein. RIN4 is thought to be a virulence target of the effectors, while the $\mathrm{R}$ proteins act as 'guards' to monitor RIN4 modification (Mackey et al. 2002, 2003). In these cases, effector recognition by RPM1 and RPS2 is indirect. An important aspect of both RPM1 and RPS2 activation is simply removal of negative regulation by RIN4 (Belkhadir et al. 2004; Day et al. 2005; Mackey et al. 2003), although further positive contributions to $\mathrm{R}$ protein activation by the effectors are possible.

In tomato (Solanum lycopersicum), recognition of the unrelated bacterial effectors AvrPto and AvrPtoB by a complex composed of Pto kinase and the NBARC-LRR protein Prf seems to be a combination of the above models (Balmuth and Rathjen 2007; Mucyn et al. 2006). Pto is the polymorphic determinant of resistance and is described formally as an $R$ gene. Pto interacts directly with both effectors in yeast through a common surface patch that overlaps the kinase catalytic cleft (Wu et al. 2004). Despite this, Prf possesses many characteristics of a typical $\mathrm{R}$ protein and also contributes to recognition of both AvrPto and AvrPtoB in plants, through an undefined mechanism (Balmuth and Rathjen 2007). Hence, activation of the Pto-Prf complex may be by a combination of direct and indirect mechanisms. Pto interacts with a novel N-terminal domain of Prf and contributes both positively and negatively to Prf regulation (Mucyn et al. 2006). Important determinants of Pto function are kinase activity, which is required for effectordependent activation but not signal transduction, and N-myristoylation, which plays an unknown role (Andriotis and Rathjen 2006; Balmuth and Rathjen 2007; de Vries et al. 2006; Rathjen et al. 1999; Wu et al. 2004). Importantly, a subset of residues within the Pto effector-binding region termed the negative regulatory patch (NRP) mediates negative regulation of signaling. Mutations within this region result in constitutive gain-offunction (CGF) forms of Pto that induce cell death in the absence of the effectors, dependent on Prf (Wu et al. 2004). How the NRP acts to restrict or promote signaling through this pathway is unknown.

Pto is a member of a small gene family that is colocated with Prf in the tomato genome (Chang et al. 2002). One homologue, Fen, mediates a Prf-dependent HR-like cell death in response to leaf treatment with the organophosphate Fenthion (Martin et al. 1994). Fen shares $80 \%$ amino acid (aa) identity 
with Pto but does not confer resistance to either AvrPto or AvrPtoB. Interestingly, Fen can elicit a Prf-dependent immune response to truncated forms of AvrPtoB lacking a C-terminal ubiquitin ligase domain (Rosebrock et al. 2007). In general, signaling by Fen is poorly understood compared with Pto. However, transient expression of Fen in the heterologous species Nicotiana benthamiana causes localized cell death that requires an $N$. benthamiana $\operatorname{Prf}(\mathrm{Nb} \operatorname{Prf})$ homologue (Chang et al. 2002). This suggests that Fen is misregulated in this species due to the absence of an unknown regulatory mechanism. Further elucidation of Fen regulation may provide insights into normal regulation of the Prf complex and control of specific immunity.

Here, we show that Fen required both kinase activity and $\mathrm{N}$ myristoylation for signaling, and interacted with the same $\mathrm{N}$ terminal domain of Prf as Pto. Strikingly, the CGF phenotype in $N$. benthamiana was suppressed by coexpression of tomato Prf, suggesting a role for Prf in regulation of Fen kinase activity. Prf can promote the phosphorylation state of both Pto and Fen, supporting the idea that Prf may influence their kinase activity. We further show that negative regulation of Prf is controlled by the previously identified Pto negative regulatory patch. Mutations within this region derepressed negative regulation of Prf by a Pto N-myristoylation mutant. Remarkably, negative regulation was also released by the effectors, dependent on Pto kinase activity. Thus, Pto holds Prf in an inactive state before activation by the effectors, and contributes both positively and negatively to Prf control. The data highlight the importance of coregulatory behavior between Prf and Pto-like kinases.

\section{RESULTS}

\section{Constitutive signaling by Fen in $N$. benthamiana requires kinase activity and $\mathrm{N}$-myristoylation.}

Overexpression of the Pto family member Fen in N. benthamiana induces an Nb Prf-dependent HR (Chang et al. 2002). To test whether overexpression was required for this phenotype, Fen was placed under control of the strong Cauliflower mosaic virus $35 \mathrm{~S}$ (35S) promoter or the weak Pto genomic promoter (Pro Pto $_{\text {) }}$ (Balmuth and Rathjen 2007; de Vries et al. 2006). The genomic Pto promoter was used in these experiments because it is partially characterized and provides a standardized low level of expression for comparison with previous results. Fen (or Pto for comparison) was expressed as a fusion protein with three carboxy-terminal HA epitopes, with or without an additional FLAG epitope (3HA or 3HAF). Transient expression of either 35S:Fen-3HA or Pro ${ }_{\text {Pto }}$ :Fen-3HAF in $N$. benthamiana leaves induced the HR within 2 to 3 days postinfiltration (dpi), whereas expression of 35S:Pto-3HAF did not (Fig. 1A). The HR induced by expression of Pro ${ }_{\mathrm{Pto}}:$ Fen-3HAF was weaker than that caused by expression of 35S:Fen-3HA. Western blotting analyses revealed much more accumulation of Fen-3HAF protein after 35S expression compared with Pro $_{\text {Pto }}$ expression. This difference probably accounts for the weaker HR phenotype resulting from Pro $_{\mathrm{Pto}}: \mathrm{Fen}-3 H A F$ expression. Importantly, the levels of Pto-3HAF accumulation resulting from $35 \mathrm{~S}$ expression were comparable with Fen-3HAF accumulation from Pro ${ }_{\text {Pto }}$ expression. Greater accumulation of Fen compared with Pto was seen throughout this study (Figs. 1 and 2), suggesting that Fen may be a more stable protein than Pto. However, Figure 1A reveals that the Fen-dependent HR in $N$. benthamiana was not due to protein overaccumulation and may, instead, be due to inappropriate regulation in this species. Two important determinants of Pto signaling activity are kinase activity and N-myristoylation (Balmuth and Rathjen 2007). To examine roles for these in constitutive signaling by
Fen, we individually mutated the codons that specify N-myristoylation (Gly-2) or kinase activity (Asp-162), respectively. Fen, Pto, fen ${ }^{G 2 A}$, or $f e n^{D 162 N}$ were expressed transiently from the $35 \mathrm{~S}$ promoter in $N$. benthamiana leaves, as genetic fusions with the FLAG tag (-F). As expected, expression of 35S:Fen-F resulted in a strong HR. No HR was visible upon expression of 35S:Pto-F or either 35S: en $^{G 2 A}-F$ or $35 \mathrm{~S}:$ fen $^{D 162 N_{-}} F$. Western blotting indicated that the lack of HR was not the result of impaired protein accumulation. Therefore, constitutive signaling by Fen in $N$. benthamiana requires both kinase activity and Nmyristoylation, similar to Pto.

\section{Tomato Prf inhibits constitutive signaling by Fen.}

We found previously that tomato Prf but not Nb Prf supported Pto-dependent recognition of avrPtoB in $N$ benthamiana (Balmuth and Rathjen 2007; Mucyn et al. 2006). This suggests a functional difference between $\mathrm{Nb}$ Prf and tomato Prf. Accordingly, a structural incompatibility between tomato Fen and $\mathrm{Nb}$ Prf leading to loss of kinase regulation may account for the ligand-independent HR observed in N. benthamiana. To test this, the Fen-dependent HR was monitored in the presence or absence of tomato Prf. In these experiments, Fen$3 H A F$ was expressed from the Pto promoter, with or without $\operatorname{Prf}$ fused to a sequence encoding five C-terminal c-myc epitopes under control of the $35 \mathrm{~S}$ promoter (35S:Prf-5myc). Prf was overexpressed here to promote formation of the Prf-Fen complex. Coexpression of Pro ${ }_{\text {Pto }}:$ Fen-3HAF with 35S:Prf5 myc abolished the Fen-dependent HR (Fig. 2A). Western blotting confirmed the presence of Fen in this experiment. In parallel, Pro $_{\mathrm{Pto}}:$ Pto-3HAF was coexpressed with and without 35S:Prf-5myc. As observed previously, coexpression of 35S:Prf with Pro $_{\text {Pto }}$ :Pto-3HAF resulted in an HR within 3 to 4 days (Mucyn et al. 2006). The HR resulting from Pto/Prf coexpression was weaker than that resulting from sole Fen expression. The data are striking because coexpression of tomato Prf with Fen or Pto leads to qualitatively different outcomes. These results concur with the observation that Fen cannot complement the requirement for Pto in the constitutive disease resistance phenotype caused by $\operatorname{Prf}$ overexpression in transgenic tomato (Mucyn et al. 2006).

Pto signaling is mediated by physical interaction with Prf (Mucyn et al. 2006). Similarly, inhibition of Fen signaling by tomato Prf may be direct. To investigate this, various Prf fragments were tested for their ability to suppress Fen signaling in $N$. benthamiana. We previously divided the 1,824-aa Prf protein into N-terminus (N-term), Solanaceous (SD), and coiledcoil (CC)-NBARC-LRR domains (Fig. 2B) (Mucyn et al. 2006).

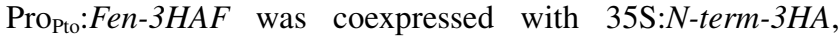
35S:SD-CC-NBARC-LRR-3HA (SCNL-3HA), or 35S:CCNBARC-LRR-3HA (CNL-3HA) in N. benthamiana, or with empty vector $(E V)$ or $35 \mathrm{~S}: \operatorname{Prf}-3 H A$ as positive and negative controls, respectively (Fig. 2C). Coexpression of Pro ${ }_{\mathrm{Pto}}:$ Fen$3 H A F$ with $35 \mathrm{~S}: P r f-3 H A, 35 \mathrm{~S}: N$-term-3HA, or 35S:CNL-3HA abolished the ligand-independent HR. The HR was not compromised when Fen was coexpressed with SCNL-3HA or EV. Western blotting analysis confirmed that all proteins were expressed in this experiment. Overall, the data show that tomato Prf suppresses constitutive Fen signaling through its Nterm or CNL moiety.

\section{Fen interacts with Prf via the N-term domain.}

To investigate a potential interaction between Fen and Prf, coimmunoprecipitation assays were performed (Fig. 3A). Protein extracts from leaves transiently coexpressing Pro ${ }_{\mathrm{Pr}}: P r f$ $5 m y c$ with Pro $_{\text {Pto }}: F e n-3 H A F$, or expressing each gene separately, were subjected to immunoprecipitation with anti-HA beads to capture Fen-3HAF (Fig. 3A). Fen was enriched in the bead 
fraction, indicating successful immunoprecipitation. Prf was detectable in the beads when Fen was present but not when the extract lacked Fen. Accordingly, immunoprecipitation of similar extracts with anti-myc beads confirmed that Fen was specifically associated with Prf (Fig. 3B). Thus, Prf and Fen interact in vivo, consistent with the idea that inhibition of Fen signaling by Prf is mediated by physical interaction. The coexpression data presented above (Fig. 2B) suggested that the $\mathrm{N}$ term or CC-NBARC-LRR domains of Prf are likely to interact with Fen. To test this, genes encoding each fragment were fused to the 5-myc sequence under control of the $35 \mathrm{~S}$ promoter, and coexpressed with $35 \mathrm{~S}:$ Fen-3HA in $N$. benthamiana leaves. Immunoprecipitation of the derived protein extracts
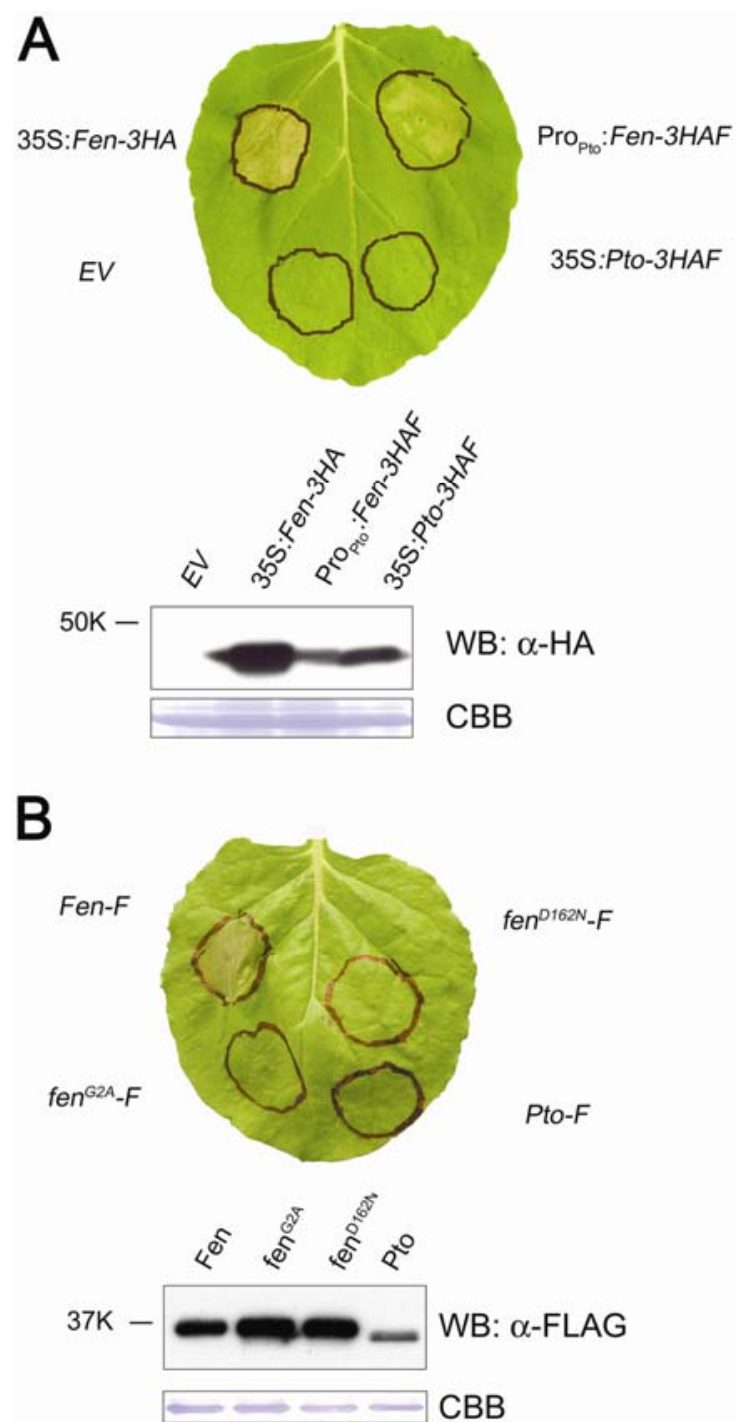

Fig. 1. Constitutive signaling by Fen in Nicotiana benthamiana requires kinase activity and $\mathrm{N}$-myristoylation. A, The Fen hypersensitive response phenotype in $N$. benthamiana does not require overexpression. $N$. benthamiana leaves were transiently transformed with Agrobacterium tumefaciens carrying 35S:Fen-3HA, Pro Pto $_{F}$ Fen-3HAF, 35S:Pto-3HAF, or empty vector $(E V)$ at an optical density at $600 \mathrm{~nm}\left(\mathrm{OD}_{600}\right)=0.5$. Picture was taken at 3 days postinfiltration (dpi). Protein expression was confirmed by Western blot using an anti-HA antibody, and equal loading verified by Coomassie Brilliant Blue (CBB) staining of the Western blot membrane. B, Fen N-myristoylation (G2A) and kinase (D162N) mutants are impaired in constitutive signaling. $N$. benthamiana leaves were transiently transformed with A. tumefaciens carrying 35S:Fen-FLAG, 35S:Fen ${ }^{G 2 A}-F L A G$,

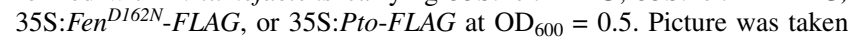
at 3 dpi. Protein expression was confirmed by Western blot using an antiFLAG antibody, and equal loading verified by CBB staining. with anti-myc beads confirmed that each Prf fragment was enriched in the bead fraction, indicating successful immunoprecipitation (Fig. 3C). Fen-3HA copurified with the N-term fragment but not the SD-CC-NBARC-LRR or CC-NBARCLRR proteins. The $\mathrm{N}$-term fragment was processed in these experiments, as observed previously (Mucyn et al. 2006). Reciprocally, we tested whether the N-term domain could be coimmunoprecipitated with Fen. Extracts containing N-term5 myc, Fen-3HAF, or both proteins together were immunoprecipitated with anti-HA beads. Western blot analysis of the immunoprecipitated fractions showed that the N-term domain was specifically associated with Fen (Fig. 3D). Thus, Fen, like Pto, interacts with Prf through its N-term domain.

\section{Prf stimulates Fen autophosphorylation.}

Pto associated with Prf undergoes a novel autophosphorylation event which is absent from uncomplexed Pto (Mucyn et al. 2006). It is unclear whether this modification represents an active form of Pto or is a maturated form associated with complex formation. The Pto/Prf complex signals constitutively after overexpression (Mucyn et al. 2006); therefore, both hypotheses are possible. In contrast, the Fen/Prf complex is inactive. To examine the phosphorylation status of Fen under conditions of constitutive signaling or repression, we examined the migration behavior of Fen on sodium dodecyl sulfate (SDS) polyacrylamide gel electrophoresis. To favor recovery of Fen/Prf complexes in these experiments, Fen was expressed from the Propto promoter while tomato Prf was overexpressed from the $35 \mathrm{~S}$ promoter. Extracts of leaf tissues expressing Fen-3HAF with or without Prf were subject to immunoprecipitation with FLAG beads, followed by elution with FLAG peptide. Similar to Pto, Fen expressed alone migrated as a single band, whereas Fen coexpressed with Prf migrated as a doublet (Fig. 4A). The slower-migrating band was removed by phosphatase treatment (Fig. 4B) and was not present in a kinase-deficient mutant, fen $^{D 162 N}$ (Fig. 4C). Thus, Fen is autophosphorylated in the presence of tomato Prf, but the autophosphorylation event is not associated with constitutive signaling. Importantly, Prf promotes autophosphorylation of both Fen and Pto.

\section{Fen contributes to regulation of Prf signaling.}

We found previously that overexpression of Prf from a dexamethasone (DEX)-inducible promoter (Dex:Prf) in N. benthamiana leaves resulted in a Pto-independent HR (Mucyn et al. 2006). This phenotype was dependent on NbSgtl and NbHsp90, consistent with activation of a disease resistance pathway. The Dex:Prf HR could be suppressed by coexpression with either $p t o^{G 2 A}$ or $p t o^{D 164 N}$, uncovering a role for Pto in negative regulation of Prf (Mucyn et al. 2006). Similarly, we tested the effect of $f e n^{G 2 A}$ or $f e n^{D 162 N}$ on the Dex:Prf-dependent HR. Dex:Prf and 35S: en $^{G 2 A}-F$ were coexpressed, or expressed individually with $E V$ as controls (Fig. 5A). Prf expression was induced 1.5 dpi by treatment with DEX. Coexpression of Dex:Prf with EV resulted in HR within $24 \mathrm{~h}$, as observed previously (Mucyn et al. 2006). However, coexpression of Dex:Prf with 35S: en $^{G 2 A}$ blocked cell death. Western blots confirmed that the absence of phenotype was not due to lack of protein accumulation. A similar experiment was conducted with $\mathrm{fen}^{\mathrm{D} 162 \mathrm{~N}}$ (Fig. 5B). Coexpression of Dex:Prf with $35 \mathrm{~S}: \mathrm{fen}^{\text {D162N }}$ did not result in the HR. Thus, similar to Pto, these experiments illustrate a role for Fen in negative regulation of Prf.

\section{The Pto NRP contributes to regulation of Prf.}

Data above and published elsewhere (Mucyn et al. 2006) show that both positive and negative interactions between Ptolike kinases and Prf are demonstrable. Positive interaction resulting in cell death is dependent on kinase activity. Negative 
regulation is less well defined. To investigate this further, we exploited the observation that the Dex:Prf-dependent HR can be suppressed in trans by coexpression of pto ${ }^{G 2 A}$ or $p^{2} o^{D 164 N}$ (Mucyn et al. 2006). Previously, we and others showed that mutations in a group of surface-exposed residues termed the NRP conferred an Nb Prf-dependent CGF phenotype to Pto (Wu et al. 2004; Xing et al. 2007). We reasoned that the NRP could be responsible for Prf regulation, and that mutations

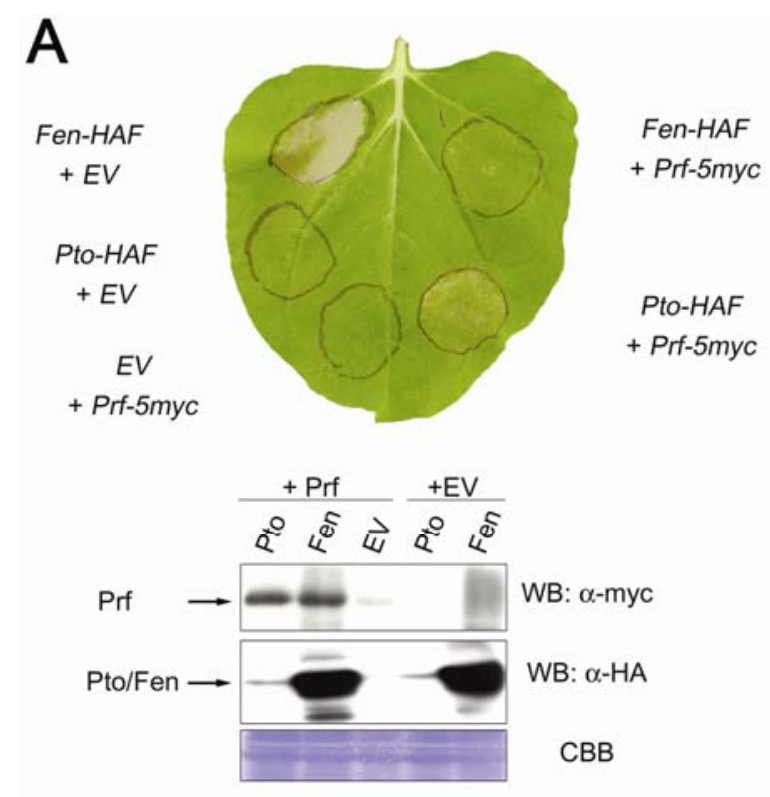

B

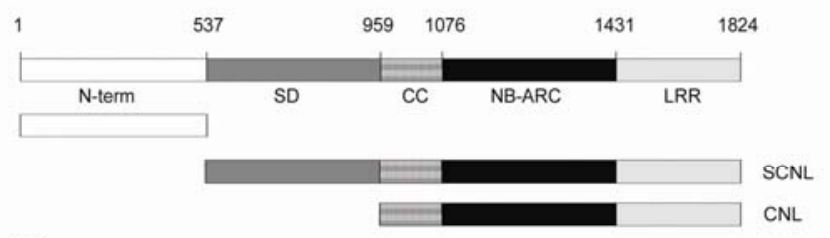

C
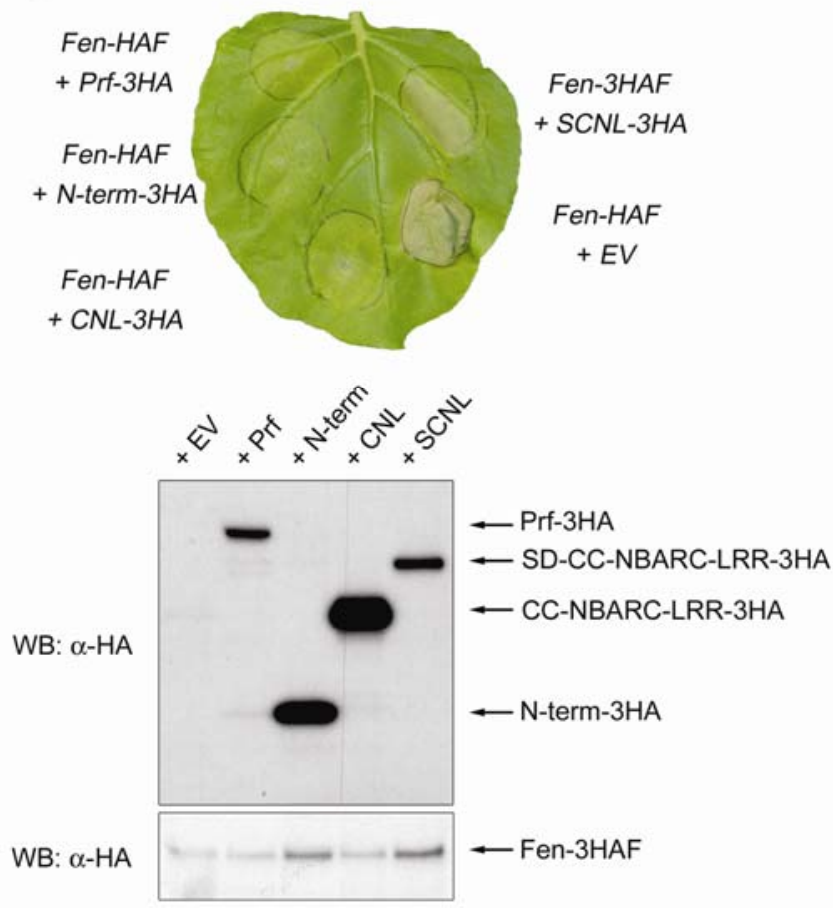

within the NRP might derepress Prf signaling. Thus, this experiment involving Dex:Prf overexpression constitutes a lossof-inhibition assay. To test a role for the Pto NRP in Prf repression, we selected three residues within the NRP (Leu-205, Ile214, and Asn-251) twinned with two residues that lie outside of but close to this region (Val-250 and Leu-295) (Fig. 6A) (Wu et al. 2004). These genes were further mutated on Gly-2 to destroy any intrinsic signaling capability (de Vries et al. 2006) and were expressed from the $35 \mathrm{~S}$ promoter as genetic fusions with the FLAG epitope sequence. None of the resulting double mutants conferred HR in $N$. benthamiana in the absence or presence of AvrPto (Supplementary Fig. 1). The double mutants pto $^{\mathrm{G} 2 \mathrm{~A}: \mathrm{L} 205 \mathrm{D}}$, pto $^{\mathrm{G} 2 \mathrm{~A}: \mathrm{I} 214 \mathrm{D}}$, and pto $^{\mathrm{G} 2 \mathrm{~A}: \mathrm{N} 251 \mathrm{D}}$ containing second-site mutations within the NRP, and pto ${ }^{\mathrm{G} 2 \mathrm{~A}: \mathrm{V} 250 \mathrm{D}}$ and pto ${ }^{\text {G2A:L295D }}$ with second-site mutations outside of this region, were tested for their ability to inhibit the Dex:Prfdependent HR. First, Dex:Prf was coexpressed with each 35S:pto double mutant, or with EV, 35S:Pto, or 35S:pto ${ }^{G 2 A}$ as controls (Fig. 6B). Coexpression of Dex:Prf with $E V$ or 35S:Pto-3HAF resulted in the HR $24 \mathrm{~h}$ after transgene induction, whereas coexpression with 35S:pto ${ }^{G 2 A}$ repressed cell death, as reported previously. Contrary to pto $^{\mathrm{G} 2 \mathrm{~A}}$, pto ${ }^{\mathrm{G} 2 \mathrm{~A}: \mathrm{L} 205 \mathrm{D}}$, pto $^{\text {G2A:I214D }}$, and pto ${ }^{\mathrm{G} 2 \mathrm{~A}: \mathrm{N} 251 \mathrm{D}}$ carrying additional mutations within the NRP were unable to inhibit Prf signaling. Western blotting confirmed accumulation of these proteins. Conversely, pto $^{\text {G2A:L295D }}$ carrying a second mutation outside of the NRP did

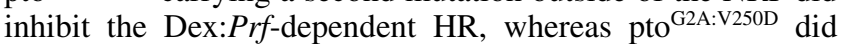
not. Western blotting revealed that the ability of these two mutants to suppress signaling correlated inversely with protein accumulation. Thus, the inability of $\mathrm{pto}^{\mathrm{G} 2 \mathrm{~A}: \mathrm{V} 250 \mathrm{D}}$ to inhibit the Dex:Prf-dependent HR may be due to low protein accumulation. Overall, mutations within the NRP abolish suppression of Prf signaling by pto ${ }^{\mathrm{G} 2 \mathrm{~A}}$.

To further assess a role for the NRP in regulation of Prf, we devised an alternate assay for activation of Prf repressed by pto $^{\mathrm{G} 2 \mathrm{~A}}$. Transient expression of 35S:Prf in N. benthamiana led to a Pto-dependent HR within 4 days (Mucyn et al. 2006). The HR was not observed when 35S:Prf was coexpressed with 35S:pto ${ }^{G 2 A}$. This result may also be explained by repression of the Prf overexpression phenotype by pto $^{\mathrm{G} 2 \mathrm{~A}}$. To test the ability of the pto double mutants to enhance signaling from 35S:Prf expression, 35S:Prf-5myc was coexpressed with each 35S:pto double mutant or with $E V, 35 \mathrm{~S}:$ Pto, or $35 \mathrm{~S}:$ pto $^{G 2 A}$ as controls. Coexpression of 35S:Prf-5myc with 35S:Pto, 35S:pto ${ }^{\text {G2A:L205D }}$,

Fig. 2. Tomato Prf suppresses the Fen constitutive gain-of-function phenotype. A, Tomato Prf abolishes constitutive Fen signaling. Pro Pto $_{\text {Fen-3HAF }}$ and 35S:Prf-5myc were coexpressed (optical density at $600 \mathrm{~nm}\left[\mathrm{OD}_{600}\right]=$ 0.4 each) or expressed individually with empty vector $(E V)$ in Nicotiana benthamiana leaves. For comparison, Pro ${ }_{\mathrm{Pto}}:$ Pto-3HAF was also expressed with 35S:Prf-5myc or EV on the same leaf. Picture was taken at 3 days postinfiltration (dpi). Protein expression was confirmed by Western blotting using an anti-myc antibody for detection of Prf and an anti-HA antibody for detection of Pto and Fen. Equal protein loading was verified by Coomassie Brilliant Blue staining. B, Subdomain diagram of the Prf protein. Numbers indicate amino acids in the derived protein sequence of Prf: $\mathrm{N}$-term, N-terminus domain; SD, Solanaceous domain; CC, coiled-coil domain; NBARC, nucleotide-binding domain shared by Apaf-1, certain R proteins, and CED-4 ATPase domain; LRR, leucine-rich repeat domain. CC-NBARC-LRR = CNL and SD-CC-NBARC-LRR = SCNL. C, Coexpression of Fen with the N-term or CNL fragments of Prf abolishes constitutive signaling. Propto:Fen-3HAF was coexpressed either with 35S:Prf5myc, 35S:CNL-3HA, 35S:SCNL-3HA, or EV in N. benthamiana leaves, using Agrobacterium tumefaciens containing each construct at $\mathrm{OD}_{600}=$ 0.4 . Picture was taken at $3 \mathrm{dpi}$. Protein expression was confirmed by Western blotting using an anti-HA antibody for detection of Prf, Prf fragments, and Fen. 
35S:pto ${ }^{\text {G2A:I214D }}$, or 35S:pto ${ }^{\text {G2A:N251D }}$ resulted in the HR (Fig. 6C), whereas coexpression with 35S:pto ${ }^{G 2 A}$ or EV did not. This suggests that mutations within the Pto NRP promote 35S:Prf signaling, despite the fact that the double mutants are incapable of signaling in themselves. In contrast, coexpression of 35S:Prf with either pto ${ }^{\mathrm{G} 2 \mathrm{~A}: \mathrm{V} 250 \mathrm{D}}$ or pto $^{\mathrm{G} 2 \mathrm{~A}: \mathrm{L} 295 \mathrm{D}}$ mutants (carrying an extra mutation outside the NRP) did not result in the HR. Western blotting confirmed the accumulation of all mutant proteins, although pto ${ }^{\text {G2A:V250D }}$ and pto ${ }^{\text {G2A:L295D }}$ accumulated to relatively low levels. However, this is unlikely to be the basis of the lack of phenotype, because coexpression of Pro $\mathrm{Pto}_{\mathrm{Pto}}$ :Pto is sufficient to stimulate 35S:Prf expression to give the HR (Mucyn et al. 2006). Remarkably, coexpression of either 35S:pto ${ }^{\text {G2A:L205D }}$, pto $^{\text {G2A:I214D }}$, or pto ${ }^{\text {G2A:N25ID }}$ with $35 \mathrm{~S}:$ Prf led to an HR that was stronger and faster than that caused by 35S:Pto/35S:Prf coexpression, suggesting that NRP mutations also contribute positively to regulation of Prf. Enhancement of Prf signaling by the pto ${ }^{\mathrm{G} 2 \mathrm{~A}}$-NRP double mutants was not dependent on their overexpression, because coexpression of 35S:Prf with Pro Pto:pto $^{\mathrm{G} 2 \mathrm{~A}: \mathrm{L} 205 \mathrm{D}}$ led to a similarly strong HR (Supplementary Fig. 2). Prf overexpression was not strictly required for this phenotype because coexpression of Pro Prf: $P r f$

with 35S:pto ${ }^{\text {G2A:L205D }}$ also resulted in a slow but significant HR. However, coexpression of Pro ${ }_{\text {Prf }}:$ Prf with Pro Pto $_{\text {pto }}$ G2A:L205D did not induce cell death, indicating that overexpression of either Prf or Pto is necessary. Phenotypes obtained for all Pto single and double mutants used in this study are summarized in Table 1. Overall, mutations within the Pto NRP promoted ligandindependent signaling by Prf, whereas mutations outside of this region did not. The data indicate that the NRP mediates negative regulation of Prf.

\section{AvrPto disrupts repression of Prf by the Pto NRP.}

The data above are consistent with a model in which Pto holds Prf in a repressed state through its NRP. The overlap between the NRP and the AvrPto/AvrPtoB interaction site on Pto suggests that effector binding could disrupt NRP-mediated negative regulation. To test this, we examined the ability of AvrPto to overcome repression of Prf by pto $^{\mathrm{G} 2 \mathrm{~A}}$, an inactive mutant that retains kinase activity and an intact NRP. For this experiment, 35S:Prf-5myc and 35S:pto ${ }^{G 2 A}-F$ were expressed separately or together in the transgenic $N$. benthamiana line 291-2, which carries a DEX-inducible avrPto-HA transgene (Chang et al. 2002). As a positive control for the HR,
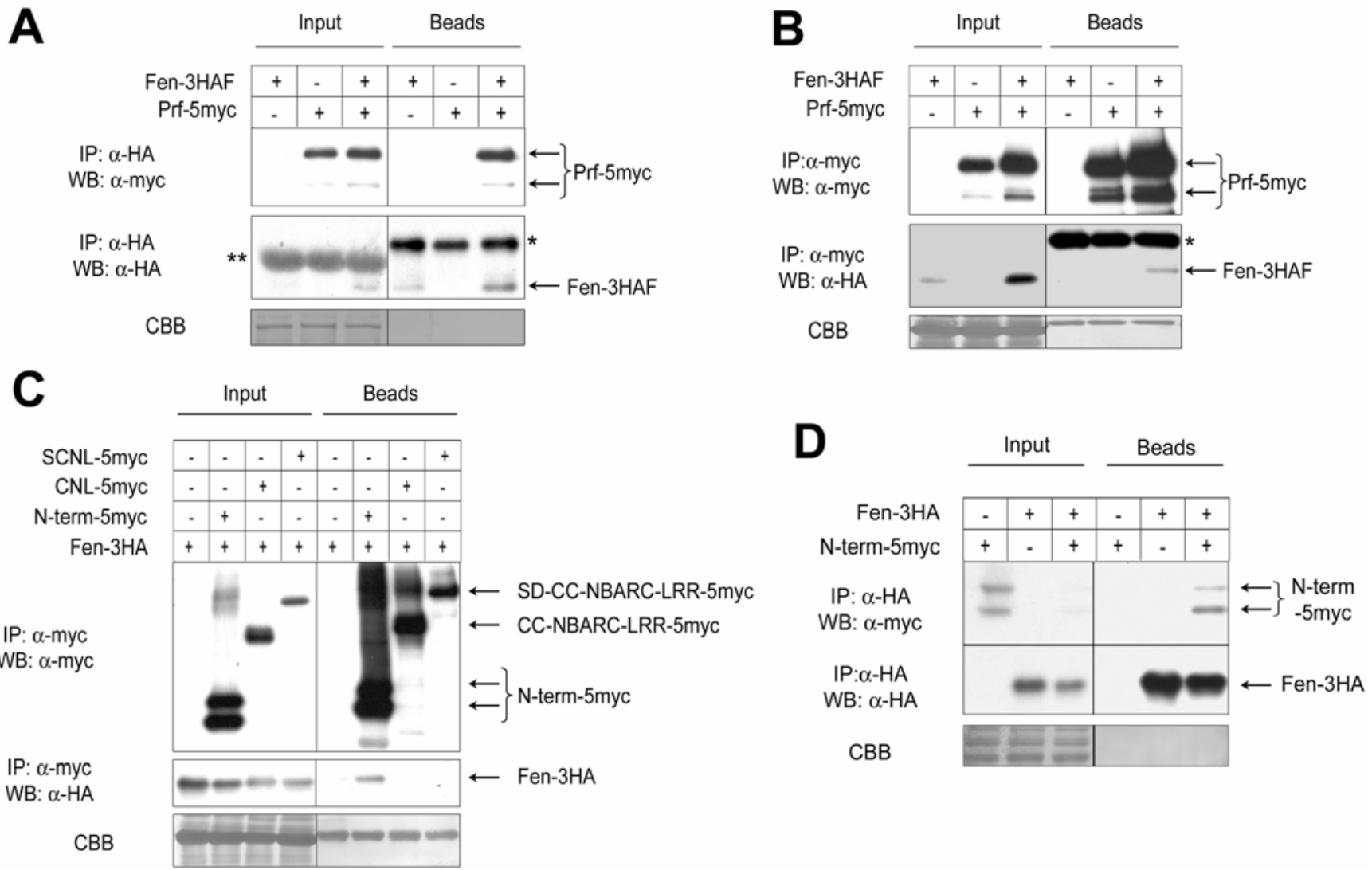

Fig. 3. Fen interacts with Prf via the N-terminus (N-term) domain. A, Prf coimmunoprecipates with Fen. Nicotiana benthamiana leaves were transiently transformed with Pro $\mathrm{Pto}_{\mathrm{t}}$ Fen-HAF, Pro $\mathrm{Prr}_{\mathrm{Pr}}$ Prf-5myc, or a 1:1 mixture of these strains. Strains for individual gene expression were balanced with Agrobacterium tumefaciens containing empty vector $(E V)$. Infiltrated leaves were harvested 2 days postinfiltration (dpi) for extraction of proteins, and protein extracts subjected to immunoprecipitation using anti-HA beads. Equal amounts by volume of crude extract (Input) and bead fractions were loaded onto the sodium dodecyl sulfate polyacrylamide gel electrophoresis (SDS-PAGE) gel. Proteins were detected by immunoblotting as indicated and equal loading confirmed by Coomassie Brilliant Blue staining; ** indicates a cross-reacting band and * a cross-reacting band corresponding to the antibody heavy chain from the beads. The experiment was performed twice with similar results. B, Fen coimmunoprecipates with Prf. $N$. benthamiana leaves were transiently transformed, and protein extracts prepared as above, except that immunoprecipitation was performed using anti-myc beads; * indicates a cross-reacting band corresponding to the antibody heavy chain from the beads. C, Fen coimmunoprecipitates with the N-term domain of Prf. 35S:Fen-3HA was coexpressed with 35S: $\mathrm{N}$-term5myc, 35S:CC-NBARC-LRR-5myc, 35S:SD-CC-NBARC-LRR-5myc, or EV. Proteins were extracted from leaves harvested 2 dpi and immunoprecipitated with anti-myc beads, followed by SDS-PAGE and immunoblotting with anti-HA or anti-myc antisera. Equivalent amounts of protein from the crude extract (Input) and bead fractions were loaded onto the SDS-PAGE gel. D, The Prf N-term domain coimmunoprecipitates with Fen. Wild-type $N$. benthamiana leaves were infiltrated with A. tumefaciens containing 35S:Fen-3HA or 35S:N-term-5myc, or a 1:1 mixture of both strains, as indicated. Protein extracts were processed as above except that immunoprecipitation was performed using anti-myc beads. These experiments were performed two times each, with similar results.

Vol. 22, No. 4, 2009 / 395 
35S:pto ${ }^{\text {G2A:L205D }}-F$ was coexpressed with 35S:Prf. Plants were treated 2 days after infiltration with DEX to induce avrPto expression or with a mock solution as a control. Individual expression of 35S:Prf, 35S:pto ${ }^{G 2 A}$, or 35S:pto ${ }^{\text {G2A:L205D }}$ did not lead to an HR, in either the presence or absence of AvrPto (Fig. 7A). Coexpression of 35S:Prf with 35S:pto ${ }^{\text {G2A:L205D }}$ induced cell death in both conditions without any obvious difference in HR timing or intensity. Coinfiltration of 35S:Prf with 35S:pto ${ }^{G 2 A}$ resulted in an HR approximately 24 to $48 \mathrm{~h}$ after induction of avrPto. Coexpression of 35S:Prf with 35S:pto ${ }^{G 2 A}$ in the absence of avrPto did not lead to cell death. Similarly, coexpression of 35S:pto ${ }^{G 2 A}$ and avrPto in the absence

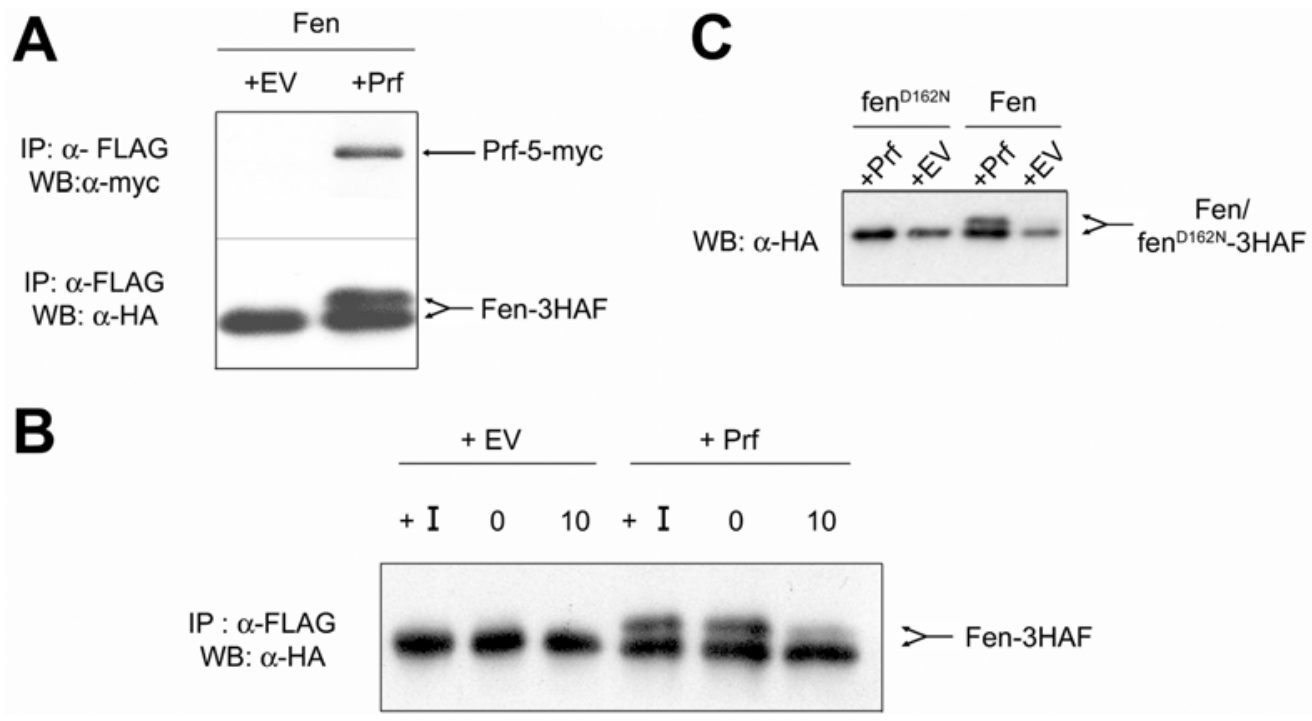

Fig. 4. Tomato Prf promotes Fen autophosphorylation. A, Altered migration of Fen in the presence of Prf. Protein extracts from Nicotiana benthamiana tissues coexpressing Pro ${ }_{\text {Pto }}: F e n-H A F$ with or without $35 \mathrm{~S}:$ Prf-5myc were subjected to immunoprecipitation using anti-FLAG beads before elution of Fen with the FLAG peptide. Elution fractions were separated by sodium dodecyl sulfate polyacrylamide gel electrophoresis (SDS-PAGE) prior to immunoblotting with anti-HA or anti-myc antisera. B, Altered migration is a result of Fen phosphorylation. Protein extracts from $N$. benthamiana tissues coexpressing Pro $_{\text {Pto }}:$ Fen-HAF with or without $35 \mathrm{~S}$ :Prf-5myc were processed as above. Eluates were subsequently treated with $\lambda$ phosphatase + phosphatase inhibitor $(+\mathrm{I})$, $\lambda$ phosphatase without subsequent incubation (0), or $\lambda$ phosphatase with incubation for $10 \mathrm{~min}$ (10). C, Altered Fen migration is a result of autophosphorylation. Extracts from tissues coexpressing Pro ${ }_{\mathrm{Pto}}$ :Fen-HAF or Pro $\mathrm{Pto}_{\mathrm{P}}: \mathrm{fen}^{D 162 N}-H A F$ with $35 \mathrm{~S}$ :Prf-5myc or empty vector $(E V)$ were subjected to purification using anti-FLAG beads as above. Eluates were resolved on a SDS-PAGE gel prior to visualization by Western blotting.
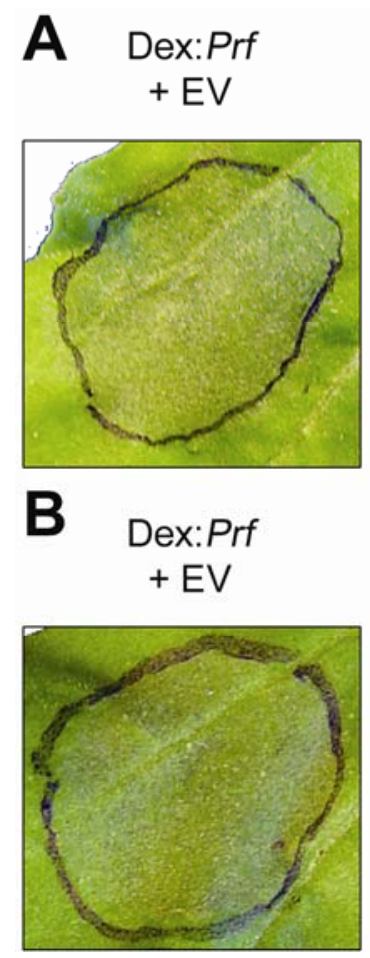

35S:fen ${ }^{62 A}-F$

$+\mathrm{EV}$
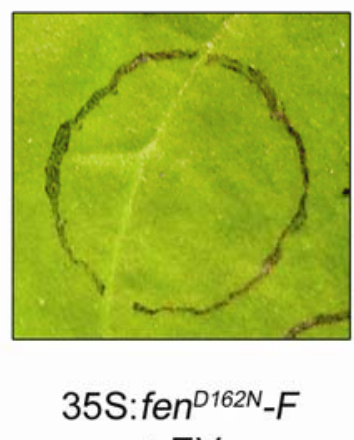

$+\mathrm{EV}$

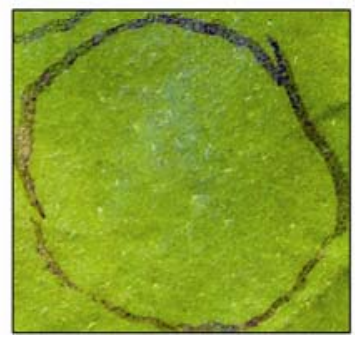

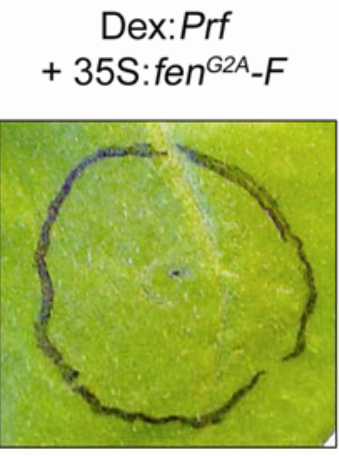

Dex:Prf $+35 S:$ fen $^{D 162 N}-F$

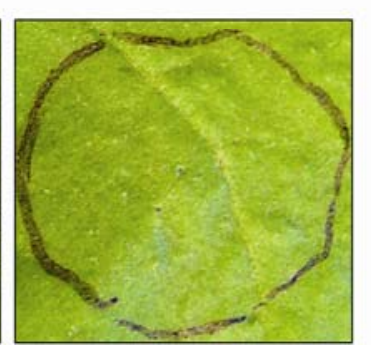

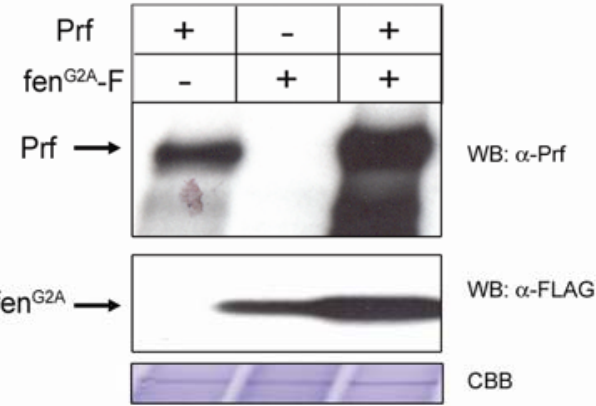

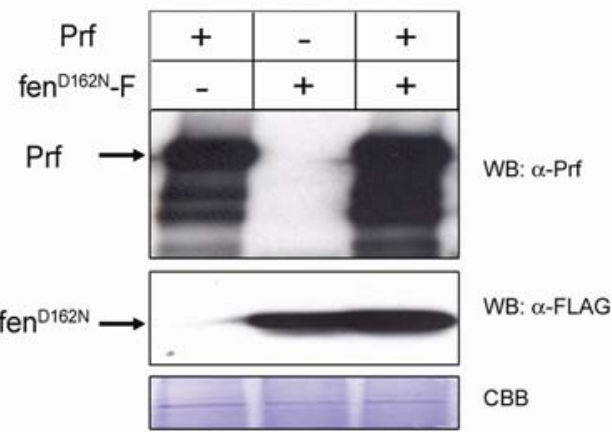

Fig. 5. Fen mutants negatively regulate Prf signaling. A, Fen ${ }^{\mathrm{G} 2 \mathrm{~A}}$ dominantly suppresses the dexamethasone (Dex):Prf hypersensitive response (HR). Dex:Prf and 35S: $\mathrm{fen}^{G 2 A}-F$ were coexpressed or expressed individually with empty vector $(E V)$ at a 1:1 ratio in Nicotiana benthamiana leaves. The leaf was treated with DEX 1.5 days postinfiltration and photographed $24 \mathrm{~h}$ after induction. Leaf tissues were harvested for protein extraction $9 \mathrm{~h}$ postinduction. Accumulation of fen ${ }^{\mathrm{G} 2 \mathrm{~A}}$ and Prf proteins was confirmed by Western blot as indicated, and equal protein loading confirmed by Coomassie staining of the Western blot membrane. B, Fen ${ }^{\text {D162N }}$ dominantly suppresses the Dex:Prf HR. Dex:Prf and $35 \mathrm{~S}: f^{2} n^{D 162 N 2}-F$ were coexpressed or expressed separately with $E V$ in $N$. benthamiana leaves. The leaf was treated and samples collected as above. 


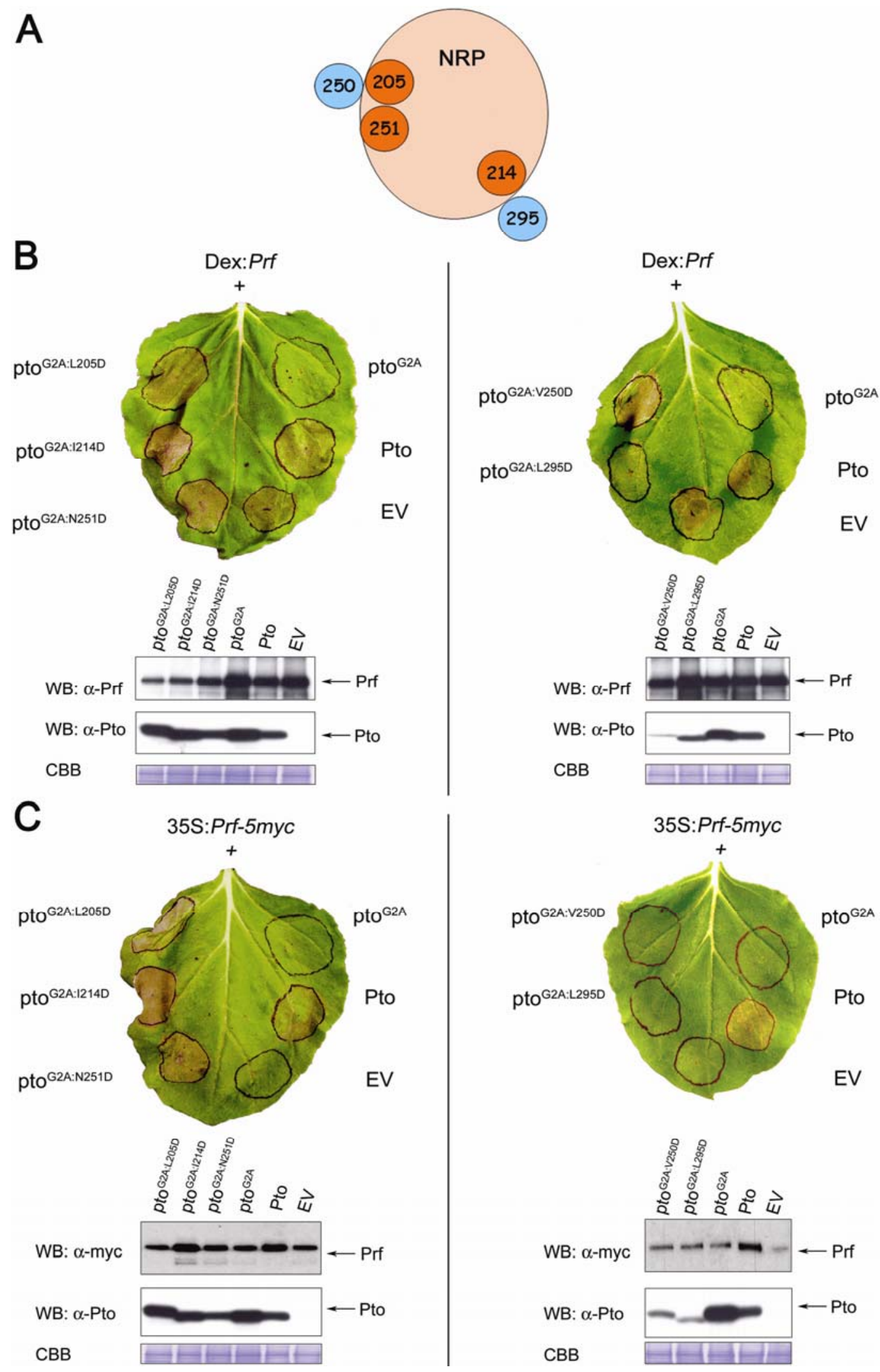

Fig. 6. Residues within the negative regulatory patch (NRP) contribute to Prf regulation. A, Schematic representation of the residues mutated in this study. Residues L205, I214, and N251 located within the NRP (represented in light orange) are colored in orange, while residues V250 and L295 outside the NRP are represented in blue. B, Pto ${ }^{\mathrm{G} A \mathrm{~A}}$ mutants carrying an additional mutation with the NRP are unable to suppress the dexamethasone (Dex):Prf-hypersensitive response. Left and right panels: Dex:Prf was coexpressed with Pto, pto mutants, or empty vector $(E V)$ as indicated. The leaf was treated with DEX 1.5 days postinfiltration and photographed $24 \mathrm{~h}$ after induction. Leaf tissues were harvested for protein extraction $9 \mathrm{~h}$ postinduction. Accumulation of fen ${ }^{\mathrm{G} 2 \mathrm{~A}}$ and Prf proteins was confirmed by Western blot as indicated, and equal protein loading confirmed by Coomassie staining of the Western blot membrane. C, Pto ${ }^{\mathrm{G} 2 \mathrm{~A}}$ mutants carrying an additional mutation with the NRP enhance Prf signaling. Left and right panels: $35 \mathrm{~S}$ :Prf- was coexpressed with Pto, pto mutants, or EV as indicated. In all cases, protein accumulation was checked by immunoblotting using the antisera as indicated. Pictures were taken at 2 days postinfiltration. 
of 35S:Prf did not induce the HR, confirming that the phenotype was a function of Prf expression. Western blotting confirmed accumulation of all proteins in this experiment. Similar experiments were performed using the kinase-deficient mutant pto $^{\mathrm{D} 164 \mathrm{~N}}$ as an alternative to pto ${ }^{\mathrm{G} 2 \mathrm{~A}}$ (Fig. 7B). In contrast to 35S:pto ${ }^{G 2 A}-F$, 35S:pto ${ }^{D 164 N}-3 H A F$ was unable to enhance 35S:Prf signaling in the presence of AvrPto. The results presented here were not specific to AvrPto, because substitution of AvrPto for AvrPtoB generated a similar dataset (Supplementary Fig. 3). In summary, negative regulation of Prf by Pto was released in the presence of the effector in a kinase-dependent manner.

\section{DISCUSSION}

Results in this article highlight important similarities and differences in coregulation of tomato Prf by the interacting kinases Pto and Fen. Fen, like Pto, requires kinase activity and $\mathrm{N}$-myristoylation for the constitutive HR in N. benthamiana. Therefore, the mode of signaling by Fen downstream of elicitation is presumed to be similar to Pto. In tomato, Fen is activated either by Fenthion treatment or by recognition of AvrPtoB derivatives lacking ubiquitination activity (Rosebrock et al. 2007). Although not tested here, it seems likely that both phenotypes would also require $\mathrm{N}$-myristoylation and kinase activity. Signaling by Fen in $N$. benthamiana is apparently effector independent. The dependence on kinase capability for constitutive signaling by Fen suggests that the phenotype is due to unregulated kinase activity. Two potential explanations for this are as follows: Fen is part of a signal transduction pathway that responds to an Agrobacterium PAMP leading to activation of Fen kinase activity, or Fen kinase activity is misregulated due to a structural incompatibility with a regulatory protein. There are currently no informative assays to measure the kinase activity of Fen or Pto during active signaling. Suppression of the Fen-dependent HR by coexpression of tomato Prf in $N$. benthamiana does not necessarily favor either hypothesis, but implicates Prf in regulation of Fen kinase activity. Previously, we concluded that constitutive signaling by the Pto/Prf complex may be due to a low-level of unregulated kinase activity (Mucyn et al. 2006). However, this is converse to the Fen-Prf example, because the outcome of Pto-Prf coexpression is enhanced signaling, not decreased, as seen for Fen-Prf coexpression in $N$. benthamiana. In addition, Fen could not complement Pto for the constitutive disease resistance phenotype caused by Prf overexpression in transgenic tomato (Mucyn et al. 2006). Therefore, there is a fundamental difference in the interaction between Prf and Pto or Fen, respectively, which results in different signaling outcomes.

Fen, like Pto, interacts with Prf through its N-term domain. It is not currently known whether Prf can bind both kinases at the same time, but it seems more likely that they use the same interaction site because they are so highly related. In this model, both Pto-Prf and Fen-Prf complexes would normally be present within the tomato cell. We found that full-length Prf and the isolated N-term domain could suppress the Fen CGF phenotype in $N$. benthamiana through physical interaction. Surprisingly, the CC-NBARC-LRR fragment could also suppress this phenotype but seemed to work through an indirect mechanism that did not include interaction with Fen. The CCNBARC-LRR fragment appears to be acting as a dominant negative form of Prf in this assay but the mechanism of inhibition is unclear. Possibly, it involves formation of an inactive complex with another protein that is required for downstream signal transduction steps. We assume that CGF signaling by Fen in $N$. benthamiana involves a complex with $\mathrm{Nb}$ Prf. In this complex, it would appear that Fen kinase activity is insufficiently controlled, whereas tomato Prf is capable of correct Fen regulation. We propose that the conflicting outcomes resulting from coexpression of particular combinations of Ptolike kinases and Prf variants is due to amino acid variations in the interaction surfaces between the protein partners. This is reminiscent of interactions between Rcr3 and $C f 2$ alleles which cause autonecrosis phenotypes in tomato (Kruger et al. 2002). $C f 2$ is the polymorphic determinant of resistance against the fungus Cladosporium fulvum expressing Avr2, whereas Rcr3 encodes a protease that is the apparent Avr2 target (Rooney et al. 2005). Autonecrosis occurs whenever lines that contain the $C f$-2 gene from $S$. pimpinellifolium are homozygous for S. lycopersicum alleles of Rcr3 (Kruger et al. 2002). In addition, two homologues of $C$. fulvum resistance gene $C f-9$ from wild tomato species caused autonecrosis when transiently expressed in $N$. benthamiana (Wulff et al. 2001). Despite the similarities of these observations to the Pto-Prf system, it is unknown whether the Rcr3 and Cf2 proteins interact.

An important difference between the constitutive signaling phenotypes of Fen in N. benthamiana and Pto variants containing a mutation in the NRP is the requirement for kinase activity. Pto CGF mutants do not require kinase activity, which suggests that they mimic the structure of the active form of Pto (Wu et al. 2004). In contrast, the Fen phenotype is kinase dependent and, thus, mimics the effector-stimulated native form of Pto. These observations suggest that the mechanisms underlying the respective CGF phenotypes must be different. Fen appears to mimic a form of Pto activated early in the elicitation process, whereas pto $^{\text {L205D }}$ mimics a terminally-activated form. The role of Pto (or Fen) kinase activity in signaling is unknown. Both Pto and Fen in complex with tomato Prf contain a novel autophosphorylation site. However, autophosphorylation is not correlated with constitutive signaling but, instead, seems to be a consequence of complex formation with Prf. Although it has usually been assumed that Pto kinase activity is required after effector binding (Rathjen et al. 1999), it is also possible that the autophosphorylated form we have detected represents a mature form of Pto that is primed for activation. In this model, the phosphorylated form would be activated by the effectors in the absence

Table 1. Activity of mutant Pto proteins in repressing or enhancing Prf-dependent signal transduction ${ }^{\mathrm{a}}$

\begin{tabular}{|c|c|c|c|c|c|c|}
\hline \multirow[b]{2}{*}{ Mutant } & \multicolumn{2}{|c|}{ Single mutant } & \multicolumn{3}{|c|}{ G2A double mutant } & \multirow[b]{2}{*}{$\begin{array}{c}\text { Negative } \\
\text { regulatory patch }\end{array}$} \\
\hline & $\begin{array}{c}\text { Constitutive } \\
\text { signaling }\end{array}$ & $\begin{array}{c}\text { AvrPto } \\
\text { recognition }\end{array}$ & $\begin{array}{l}\text { Constitutive } \\
\text { signaling }\end{array}$ & $\begin{array}{l}\text { Inhibition of } \\
\text { Dex:Prf-HR }\end{array}$ & $\begin{array}{l}\text { Enhancement of } \\
\text { 35S:Prf signaling }\end{array}$ & \\
\hline L205D & + & ND & - & - & + & + \\
\hline $\mathrm{I} 214 \mathrm{D}$ & + & ND & - & - & + & + \\
\hline V250D & - & + & - & ${ }^{\mathrm{b}}$ & - & - \\
\hline N251D & + & ND & - & - & + & + \\
\hline L295D & - & + & - & + & - & - \\
\hline G2A & - & - & - & + & - & - \\
\hline
\end{tabular}

${ }^{\mathrm{a}} \mathrm{ND}=$ not determined.

${ }^{\mathrm{b}}$ Lack of inhibition probably related to poor accumulation of the mutant protein. 

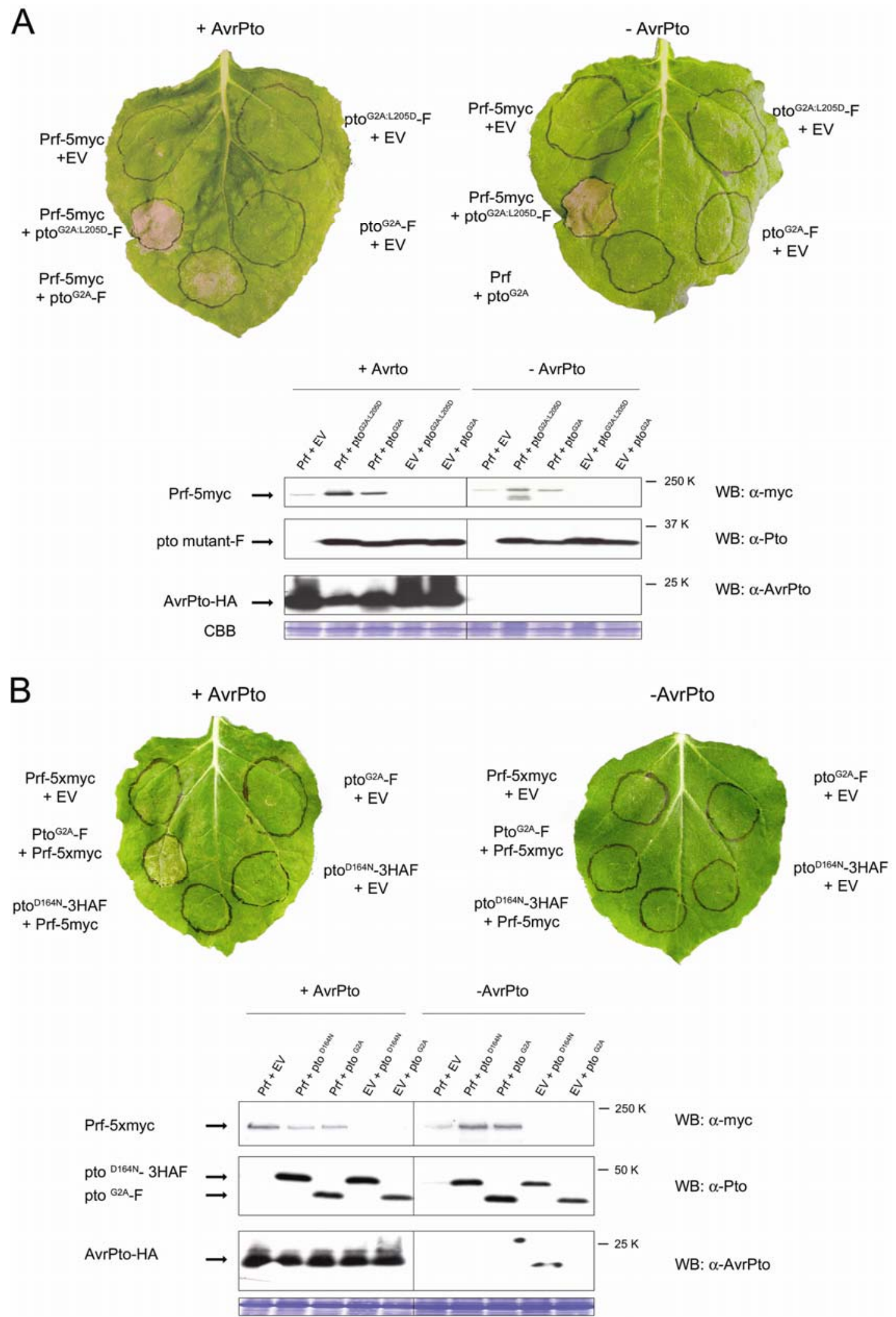

Fig. 7. AvrPto disrupts negative regulatory patch-mediated negative regulation of Prf. A, 35S:Prf-5myc was co-expressed with 35S:pto ${ }^{G 2 A}-F$ or 35S:pto ${ }^{\text {G2A:L205D }}-F$ at a 1:1 ratio in transgenic Nicotiana benthamiana line 291-2 (Dex:avrPto-HA) plants. Each construct was also expressed individually, balanced with Agrobacterium tumefaciens carrying empty vector $(E V)$. Transformed leaves were treated with dexamethasone (+AvrPto) at 2 days postinfiltration to induce AvrPto expression, or a mock solution (-AvrPto) as control. Protein accumulation was checked by immunoblotting using the antisera as indicated. B, Disruption of negative regulation by AvrPto requires Pto kinase activity. Agrobacterium carrying 35S:Prf-5myc at optical density at $600 \mathrm{~nm}=0.4$ was co-infiltrated with strains carrying $35 \mathrm{~S}:$ pto $^{G 2 A}-F$ or $35 \mathrm{~S}:$ pto $^{\text {D164N }}-3 H A F$ at a $1: 1$ ratio in transgenic N. benthamiana line $291-2$ (Dex:avrPto-HA). Each construct was also expressed individually, balanced with Agrobacterium carrying EV at a 1:1 ratio. Transformed leaves were treated as above and protein accumulation checked by immunoblotting as indicated. 
of a further requirement for kinase activity. Identification of the novel phosphoresidue or phosphoresidues will provide further insight regarding its role in effector recognition and response and allow testing of the proposed model.

Pto or Fen mutants lacking N-myristoylation or kinase activity are able to suppress constitutive Prf signaling due to overexpression. The inhibition of Prf by these mutants underlines the ability of both kinases to negatively regulate Prf, most likely by direct interaction as Pto and N-term interact in yeast (V. Ntoukakis and J. P. Rathjen, unpublished data). We previously identified the NRP as an important area on the Pto surface that controls many aspects of signaling (Wu et al. 2004), and this was subsequently confirmed by high-resolution structural analysis (Xing et al. 2007). Most importantly, mutations within this region conferred Prf-dependent CGF activity. Data in this article are consistent with a new model in which the Pto NRP normally acts to suppress Prf signaling through a direct interaction. Nevertheless, it remains to be demonstrated the NRP itself contacts Prf. This second point is important, because the NRP lies within a broader area required for interaction with AvrPto and AvrPtoB. Therefore, a model in which the NRP contacted Prf directly would need to explain how the effectors could target the largely hidden binding site. Given this model, the effector would likely perturb the interaction between Pto and Prf without dissolving the complex, consistent with experimental observations (Mucyn et al. 2006). Conversely, in an indirect model in which NRP residues do not contact Prf, the NRP and effector binding sites would be exposed to the cellular milieu. This model explains how the effector is able to access the catalytic cleft of the kinase but not how changes at the NRP are able to influence Prf activity. Finally, intermediate models in which only part of the NRP contacts Prf are possible, potentially allowing both effector access and regulation of kinase activity.

Mutations within the NRP derepressed Prf inhibition by pto $^{\text {G2A }}$. In these experiments, both Pto and Prf were overexpressed, which leads to significantly enhanced levels of the Pto-Prf complex. Within the complex, the expected constitutive signaling activity was evidently repressed by the Pto G2A mutation, which abolishes $\mathrm{N}$-myristoylation. In turn, the complex was derepressed by coexpression of the effectors or mutations within the NRP. Thus, the normal requirement for Pto Nmyristoylation was complemented by overexpression of the complex components. What is the basis of this complementation? The requirement for Pto $\mathrm{N}$-myristoylation was previously overcome by overexpression of pto ${ }^{\mathrm{G} 2 \mathrm{~A}}$ in transgenic tomato (Balmuth and Rathjen 2007; Loh et al. 1998;). The role of Nmyristoylation in signaling by the Pto/Prf complex is not known but is most likely responsible for membrane localization (Balmuth and Rathjen 2007). However, we cannot rule out alternative roles for $\mathrm{N}$-myristoylation; for example, in enhancing protein-protein interactions, which is also a predictable outcome of complex overexpression. The second point of $\mathrm{Pto} / \mathrm{Prf}$ regulation is clearly Pto kinase activity. According to existing models, Pto is normally in a repressed state, which is derepressed by interaction with effectors. Repression may involve points of contacts with Prf as discussed above or the myristoylated N-terminus, or both. Thus, perturbation of the NRP by the effectors could derepress Pto kinase activity, resulting in regulatory phosphorylation. We propose a two-step model for activation. First, Prf is held in an inactive form by Pto mediated by the NRP. After effector perception, Pto kinase activity "fires" to release negative regulation, which can be mimicked by NRP mutation. This, in turn, releases the Pto Nmyristoyl moiety, which is probably required for membrane localization. It is also possible that the requirement for Pto Nmyristoylation precedes the role of regulatory kinase activity.
Xing and associates (2007) have suggested that the role of Pto kinase activity is limited to stabilization of the activation segment to allow interaction with the effector, but this model does not explain why pto $^{\mathrm{D} 164 \mathrm{~N}}$ is not activated by AvrPto. Overall, the data suggest a very complex interaction between the Pto NRP and Prf, in which signaling is coordinated through mutual repression, regulation of kinase activity, and conditional exposure of the myristoylated Pto N-terminus.

Activation of defense responses by effector-mediated disruption of negative regulation may be a general feature of complexes containing Prf and Pto-like kinases in tomato. Most residues composing the Pto NRP are chemically conserved or identical in Fen (data not shown). Thus, the mechanism or mechanisms underlying negative regulation of Prf by Fen are probably highly related to that mediated by Pto. We predict that mutation of the cognate residues in Fen would result in constitutive, kinase-independent signaling in tomato. In the context of effector-mediated activation, it will be important to determine which residues of Fen mediate interaction with those AvrPtoB derivatives lacking E3 ligase activity. Potentially, Prf may be involved in similar coregulatory interactions, with the remaining Pto-like kinases comprising Pth2, Pth3, and Pth4. Each of these complexes could provide recognition of yet unknown pathogen elicitors.

Activation of NBARC-LRR proteins by effector-mediated removal of negative regulation may be a widespread phenomenon, as is observed in the RIN4/RPS2 system. Arabidopsis RIN4 is a negative regulator of RPS2 signaling in the absence of pathogen (Day et al. 2005; Mackey et al. 2003). Degradation of RIN4 by the bacterial effector protease AvrRpt2 releases negative regulation of RPS2, leading to activation of plant defenses. In contrast to Pto, a positive role for RIN4 in RPS2 activation has not been described. RIN4 is also a negative regulator of RPM1 signaling, although the Arabidopsis rin4, rps 2 mutant is not embryo lethal, indicating that removal of negative regulation may not be sufficient for full activation of RPM1 (Belkhadir et al. 2004). In this line, partial activation of RPM1 may be a consequence of limited accumulation in the absence of RIN4. This is similar to observations with Pto and Prf, which are mutually required for optimal accumulation. Both AvrRpm1 and AvrB trigger phosphorylation of RIN4 which is correlated with RPM1 activation (Mackey et al. 2002; Nimchuk et al. 2007). However, the mechanisms of RIN4 phosphorylation and roles of phosphorylation in RPM1 activation remain unknown. It will be intriguing to see whether the phosphorylated RIN4 residues are required for negative regulation of RPM1. Finally, it remains to be determined how apparent targeting of Pto and RIN4 by bacterial effectors is part of a plausible strategy for virulence.

\section{MATERIALS AND METHODS}

\section{Cloning of fen and pto mutants.}

Pto and Fen mutants were generated and cloned into the binary vector pTFS-40 as described (de Vries et al. 2006), except for the pto ${ }^{\mathrm{G} 2 \mathrm{~A}}$ NRP double mutants, which were cloned into pCB302-3 as described (Wu et al. 2004).

\section{Transient gene expression.}

Growth and transient expression conditions for $N$. benthamiana were as described (Wu et al. 2004) using the Agrobacterium tumefaciens C58C1. For induction of avrPto expression in the transgenic line 291-2 containing Dex:avrPto (Chang et al. 2002) or of Prf during transient assays with Dex:Prf, leaves were painted with $30 \mu \mathrm{M}$ dexamethasone (Sigma, St. Louis) in aqueous solution supplemented with $0.1 \%$ (vol/vol) Tween-20 (Sigma). 


\section{Protein analysis.}

For the analysis of protein accumulation in planta, leaf samples were ground in liquid nitrogen and solubilized in $1 \times$ SDS loading buffer (Laemmli 1970). Proteins were detected according to the target epitope with anti-HA antibody, clone 3F10 (Roche, Branchburg, NJ, U.S.A.); anti-c-myc, clone A14 (Santa Cruz); or anti-FLAG antibody, clone M2 (Sigma), Prf-peptide antibody (Mucyn et al. 2006), and Pto-antibody (Wu et al. 2004). Immunocomplexes were labeled with a peroxidase-conjugated secondary antibody (anti-rabbit, anti-rat [Sigma], or anti-mouse [DAKO]) as appropriate and detected using the ECL reagent (GE Healthcare, Chalfont St. Giles, U.K.).

\section{Immunoprecipitation and coimmunoprecipitation assays.}

Protein extracts from $N$. benthamiana were prepared in extraction buffer (50 mM Tris- $\mathrm{HCl}, \mathrm{pH} 7.5 ; 150 \mathrm{mM} \mathrm{NaCl} ; 5$ mM EDTA; 2 mM dithiothreitol [DTT]; 10\% glycerol; and 1\% polyvinyl polypyrrolidone [PVPP] supplemented with a plant protease inhibitor cocktail [Sigma]). Extracts were centrifuged at $15,000 \times g$ for $10 \mathrm{~min}$ at $4^{\circ} \mathrm{C}$. Supernatants were either subjected to ultracentrifugation at $100,000 \times g$ for $20 \mathrm{~min}$ at $4^{\circ} \mathrm{C}$ or passed through $0.45-\mu \mathrm{m}$ filters to remove particulate material. Extracts were incubated with anti-HA affinity matrix clone 3F10 (Roche), anti-myc beads clone 9E10 (Santo Cruz), or anti-FLAG M2-agarose gel (Sigma), as indicated in figure legends. Prior to use, affinity matrices were blocked with $1 \%$ bovine serum albumin. Extracts were mixed with affinity matrices for $2 \mathrm{~h}$ at $4^{\circ} \mathrm{C}$, with constant rotation to ensure mixing. Affinity matrixes were washed four times with $1 \mathrm{ml}$ of extraction buffer containing $250 \mathrm{mM} \mathrm{NaCl}$. Proteins were stripped from the bead fraction by boiling in SDS loading buffer. Elution from anti-FLAG beads was performed by incubating antiFLAG M2 affinity gel with equilibration buffer containing $10 \%$ glycerol and FLAG peptide (Sigma) at $200 \mu \mathrm{g} / \mathrm{ml}$ for 10 min at $25^{\circ} \mathrm{C}$ with agitation.

\section{Dephosphorylation assays.}

Dephosphorylation assays were performed as described by Mucyn and associates (2006). A phosphorylation-induced doublet was reliably detected by Western blot after separation on $8 \%$ SDS-polyacrylamide gels run at $110 \mathrm{~V}$.

\section{ACKNOWLEDGMENTS}

We thank V. Ntoukakis and J. Gutierrez-Pulgar for comments on the manuscript. The support of the Gatsby Charitable Foundation is gratefully acknowledged.

\section{LITERATURE CITED}

Andriotis, V. M., and Rathjen, J. P. 2006. The Pto kinase of tomato, which regulates plant immunity, is repressed by its myristoylated $\mathrm{N}$ terminus. J. Biol. Chem. 281:26578-26586.

Balmuth, A., and Rathjen, J. P. 2007. Genetic and molecular requirements for function of the Pto/Prf effector recognition complex in tomato and Nicotiana benthamiana. Plant J. 51:978-990.

Belkhadir, Y., Nimchuk, Z., Hubert, D. A., Mackey, D., and Dangl, J. L. 2004. Arabidopsis RIN4 negatively regulates disease resistance medi- ated by RPS 2 and RPM1 downstream or independent of the NDR1 signal modulator and is not required for the virulence functions of bacterial type III effectors AvrRpt2 or AvrRpm1. Plant Cell16:2822-2835.

Chang, J. H., Tai, Y. S., Bernal, A. J., Lavelle, D. T., Staskawicz, B. J., and Michelmore, R. W. 2002. Functional analyses of the Pto resistance gene family in tomato and the identification of a minor resistance determinant in a susceptible haplotype. Mol. Plant-Microbe Interact. 15:281-291.

Chisholm, S. T., Coaker, G. T., Day, B., and Staskawicz, B. J. 2006. Hostmicrobe interactions: Shaping the evolution of the plant immune response. Cell 124:803-814.

Day, B., Dahlbeck, D., Huang, J., Chisholm, S. T., Li, D., and Staskawicz, B. J. 2005. Molecular basis for the RIN4 negative regulation of RPS2 disease resistance. Plant Cell 17:1292-1305.

de Vries, J. S., Andriotis, V. M. E., Wu, A.-J., and Rathjen, J. P. 2006. Tomato Pto encodes a functional $\mathrm{N}$-myristoylation motif that is required for signal transduction in Nicotiana benthamiana. Plant J. 45:31-45.

Jones, J. D., and Dangl, J. L. 2006. The plant immune system. Nature 444:323-329.

Kruger, J., Thomas, C. M., Golstein, C., Dixon, M. S., Smoker, M., Tang, S., Mulder, L., and Jones, J. D. G. 2002. A tomato cysteine protease required for $\mathrm{C} f$-2-dependent disease resistance and suppression of autonecrosis. Science 296:744-747.

Laemmli, U. K. 1970. Cleavage of structural proteins during the assembly of the head of bacteriophage T4. Nature 227:680-685.

Loh, Y. T., Zhou, J., and Martin, G. B. 1998. The myristylation motif of Pto is not required for disease resistance. Mol. Plant-Microbe Interact. 11:572-576.

Mackey, D., Holt, B. F., Wiig, A., and Dangl, J. L. 2002. RIN4 interacts with Pseudomonas syringae type III effector molecules and is required for RPM1-mediated resistance in Arabidopsis. Cell 108:743-754.

Mackey, D., Belkhadir, Y., Alonso, J. M., Ecker, J. R., and Dangl, J. 2003. Arabidopsis RIN4 is a target of the type III virulence effector AvrRpt2 and modulates RPS2-mediated resistance. Cell 112:379-389.

Martin, G. B., Frary, A., Wu, T., Brommonschenkel, S., Chunwongse, J., Earle, E. D., and Tanksley, S. D. 1994. A member of the tomato Pto gene family confers sensitivity to fenthion resulting in rapid cell death. Plant Cell 6:1543-1552.

Mucyn, T. S., Clemente, A., Andriotis, V. M., Balmuth, A. L., Oldroyd, G. E., Staskawicz, B. J., and Rathjen, J. P. 2006. The tomato NBARC-LRR protein Prf interacts with Pto kinase in vivo to regulate specific plant immunity. Plant Cell 18:2792-2806.

Nimchuk, Z. L., Fisher, E. J., Desveaux, D., Chang, J. H., and Dangl, J. L. 2007. The HopX (AvrPphE) family of Pseudomonas syringae type III effectors require a catalytic triad and a novel $\mathrm{N}$-terminal domain for function. Mol. Plant-Microbe Interact. 20:346-357.

Rathjen, J. P., Chang, J. H., Staskawicz, B. J., and Michelmore, R. W. 1999. Constitutively active Pto induces a Prf-dependent hypersensitive response in the absence of avrPto. EMBO (Eur. Mol. Biol. Organ.) J. 18:3232-3240.

Rooney, H. C. E., van't Klooster, J. W., van der Hoorn, R. A. L., Joosten, M. H. A. J., Jones, J. D. G., and de Wit, P. J. G. M. 2005. Cladosporium Avr2 inhibits tomato Rcr3 protease required for Cf-2-dependent disease resistance. Science 308:1783-1786.

Rosebrock, T. R., Zeng, L., Brady, J. J., Abramovitch, R. B., Xiao, F., and Martin, G.B. 2007. A bacterial E3 ubiquitin ligase targets a host protein kinase to disrupt plant immunity. Nature 448:370-374.

Wu, A.-J., Andriotis, V. M. E., Durrant, M. C., and Rathjen, J. P. 2004. A patch of surface-exposed residues mediates negative regulation of immune signaling by tomato Pto kinase. Plant Cell 16:2809-2821.

Wulff, B. B. H., Thomas, C. M., Smoker, M., Grant, M., and Jones, J. D. G. 2001. Domain swapping and gene shuffling identify sequences required for induction of an Avr-dependent hypersensitive response by the tomato Cf-4 and Cf-9 proteins. Plant Cell 13:255-272.

Xing, W., Zou, Y., Liu, Q., Liu, J., Luo, X., Huang, Q., Chen, S., Zhu, L., Bi, R., Hao, Q., Wu, J. W., Zhou, J. M., and Chai, J. 2007. The structural basis for activation of plant immunity by bacterial effector protein AvrPto. Nature 449:243-247. 